\title{
Labs urged to pre-empt bioterrorism threat
}

\section{Erika Check, Washington}

An initiative is under way to get biotechnology companies to reduce the risks that their staff or labs could nurture bioterrorism — either by accident or by design.

Two non-profit groups, the International Institute for Strategic Studies (IISS) and the Chemical and Biological Arms Control Institute, announced the initiative in Washington on 20 February.

They are hoping that pharmaceutical and biotechnology companies will voluntarily join a scheme to prevent the misuse of tools at their researchers' disposal.

Michael Moodie, president of the arms control institute, and IISS president Terence Taylor are drawing up a charter for companies to sign certifying that they have taken steps to address bioterrorism risks. They also plan to bring industry leaders, academic scientists and government officials together at forums to discuss the issue.

Taylor says that the initiative is inspired by a report released last October by a National Academy of Sciences panel. The panel, chaired by Gerald Fink, a geneticist at the Whitehead Institute in Cambridge, Massachusetts, recommended steps to prevent the misuse of biological knowledge (see Nature 425, 647; 2003). The Bush administration initially said that it would respond to the report within a month, but hasn't yet done so.

Government officials welcomed Taylor and Moodie's plan, saying that they have few

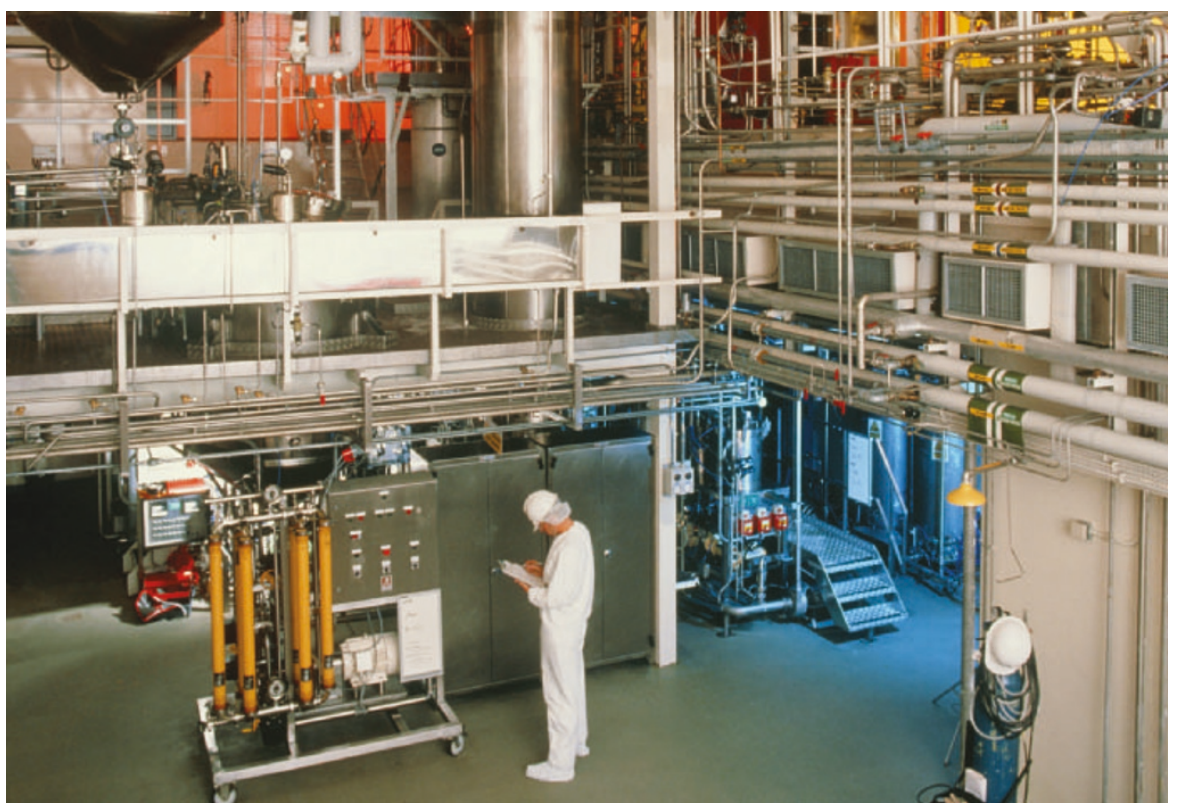

Risk of abuse? A large-scale fermenter used in production of monoclonal antibodies for vaccines.

mechanisms for keeping track of drugindustry labs. "Now that the biological weapons protocol is dead, we have no interactions with those communities," says Jennie Gromoll, an official handling biological weapons issues at the state department.

Others cautioned that it might be difficult for the initiative to draw a response from the private sector. Eileen Choffnes, an armscontrol expert at the national academy, says that many companies aren't convinced that their work could contribute to bioterrorism. "Unless you can define the problem with objective evidence, many people are just going to go through the motions," she says.

Una Ryan, chief executive of Avant in Needham, Massachusetts, which is working on developing an anthrax vaccine, supports the move "because there's no agency constantly monitoring the situation".

\section{Electron movements pinned down to the split second}

Mark Peplow, London

Physicists in Austria say they that have observed events separated by the shortest time interval ever, and plan to use the technique to study atomic phenomena.

A group led by Ferenc Krausz of Vienna University of Technology used pulses of laser light to watch electrons moving around atoms, and were able to distinguish events that took place 100 attoseconds - or $10^{-16}$ seconds - apart (see page 817 of this issue).

Krausz and his team say that the research could improve understanding of the role of electrons in superconductivity and in giant magnetoresistance, a magnetic effect used in data-storage devices. "It's only on the attosecond timescale that you see these things happening," says Roger Falcone, a physicist at the University of California, Berkeley.

The attosecond $\left(10^{-18} \mathrm{~s}\right)$, also known as a quintillionth of a second, is the timescale of atomic events. In Niels Bohr's 1913 model of a hydrogen atom, it takes about 150 attoseconds for an electron to orbit the proton.

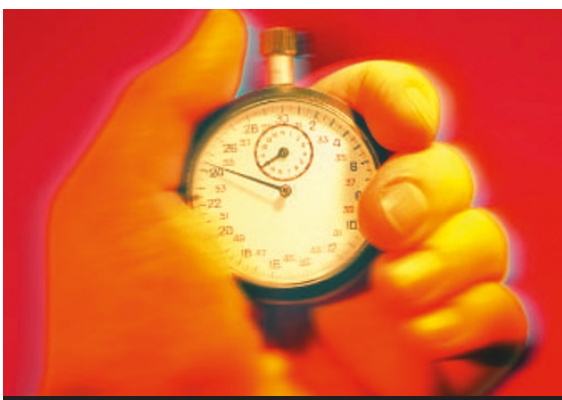

Just a tick: scientists are using ever-shorter timescales to investigate chemical reactions.

Krausz used ultraviolet-light pulses of 250-attosecond duration to excite electrons in atoms of neon, raising them to a higher energy level. These electrons come out of the atoms with a range of different momenta. A second pulse of light, delivered just after the first, then gives the electrons an extra jolt. Changing the delay between these two pulses produces a different distribution of momentum values for the electrons, which the scientists measured with a sensitive electron detector called a 'streak camera'. Comparing these distributions gave the team a snapshot of how the electrons differed when hit with an early or late second pulse.

Krausz thinks that the technique could soon be refined to track events a few tens of attoseconds apart, telling physicists how electrons rearrange themselves inside an atom when they move between different orbits.

Recent progress in using fleeting laser flashes to study short time scales has opened up the field of femtochemistry, in which the motion of atoms and molecules is tracked on the femtosecond $\left(10^{-15} \mathrm{~s}\right)$ timescale, to see how they rearrange themselves during chemical reactions. Probing even shorter timescales could provide more insight.

"Every time we get shorter pulses, chemists say that we don't really need them," says David Klug, a photochemist at Imperial College, London. "But then they find a way to use them. Once it becomes convenient, people will find applications for it." 